\title{
Erratum to: Evaluation of Anti-Inflammatory Effects of Helminthostachys zeylanica Extracts via Inhibiting Bradykinin-Induced MMP-9 Expression in Brain Astrocytes
}

\author{
Chuen-Mao Yang ${ }^{1,2,3}$ • Sien-Hung Yang ${ }^{4}$ Tsong-Hai Lee ${ }^{5}$. Jia-You Fang ${ }^{3}$. \\ Chwan-Fwu Lin ${ }^{6}$ - Mei-Jie Jou ${ }^{1} \cdot$ Hsi-Lung Hsieh ${ }^{5,7}$
}

Published online: 25 April 2016

(C) Springer Science+Business Media New York 2016

\section{Erratum to: Mol Neurobiol}

DOI 10.1007/s12035-015-9511-9

The authors would like to publish this correction note, for this article unfortunately missed some important contributors, affiliations and grant support.

Prof. Chuen-Mao Yang, all the experiments were performed at his laboratory and he also designed the experiments and supervised the technicians to conduct the research work. Moreover, he supplied the reagents, apparatus and technical assistance in the study.

Prof. Mei-Jie Jou, who provided the invaluable cell line RBA-1 cells for this paper and also provided important advice during the process of experiments.

The online version of the original article can be found at http://dx.doi.org/ 10.1007/s12035-015-9511-9.

Hsi-Lung Hsieh

hlhsieh@mail.cgust.edu.tw

1 Department of Physiology and Pharmacology and Health Ageing Research Center, College of Medicine, Chang Gung University, Kwei-San Tao-Yuan, Taiwan

2 Department of Anesthetics, Chang Gung Memorial Hospital at Lin-Kou and College of Medicine, Chang Gung University, Kwei-San Tao-Yuan, Taiwan

3 Research Center for Industry of Human Ecology and Graduate Institute of Health Industry Technology, Chang Gung University of Science and Technology, Kwei-San Tao-Yuan, Taiwan
Furthermore, Acknowledgments statement should be included for Chuen-Mao Yang's support to state "This work was also supported by the Ministry of Education, Taiwan, Grant number: EMRPD1E1641; the Ministry of Science and Technology, Taiwan, Grant number: MOST 104-2320-B182-010; Chang Gung Medical Research Foundation, Grant number: CMRPD1C0103, CMRPD1F0021, CMRPD1F0022.”

All authors have signed and agreed with these changes in the sequence of authorship and with the additional statement under Acknowledgments as given above.

4 Department of Traditional Chinese Medicine, Chang Gung Memorial Hospital at Taipei and School of Traditional Chinese Medicine, College of Medicine, Chang Gung University, Gui-Shan Tao-Yuan, Taiwan

5 Stroke Center and Stroke Section, Department of Neurology, Chang Gung Memorial Hospital, Linkou Medical Center and College of Medicine, Chang Gung University, Tao-Yuan, Taiwan

6 Department of Cosmetic Science, Chang Gung University of Science and Technology, Gui-Shan Tao-Yuan, Taiwan

7 Department of Nursing, Division of Basic Medical Sciences, and Research Center for Industry of Human Ecology, Chang Gung University of Science and Technology, Gui-Shan Tao-Yuan, Taiwan 